\title{
Effect of an individualised training programme during weight reduction on body composition: a randomised trial
}

\author{
Josef Schwingshandl, Karl Sudi, Brigitte Eibl, Silvia Wallner, Martin Borkenstein
}

\begin{abstract}
Objective-To study the effect of a standardised training programme focusing on maintenance of fat free mass during weight reduction by energy reduction in obese children.

Design-Randomised trial of physical training programme and dietary advice (group A) versus dietary advice alone (group B).

Subjects-Thirty obese children and adolescents (14 group A, 16 group B) participated in the 12 week long programme; 20 children (10 group $A, 10$ group $B$ ) were also reassessed after one year.

Measurements-Fat free mass was estimated from the resistance index, obtained by bioelectrical impedance analysis at baseline, after four, eight, and 12 weeks in all subjects, and after one year in 20 subjects.

Results-The mean (SD) change in fat free mass was significantly different between the two groups after 12 weeks (group A, 2.68 (3.74) kg; group B, 0.43 $(1.65) \mathrm{kg})$. The change in body weight after one year was inversely correlated with the change in fat free mass after 12 weeks $(r=-0.44)$, as assessed in the 20 subjects. Conclusions-A standardised training programme as used in this study can prevent reduction in fat free mass during weight loss in obese children. Reduction in fat free mass during weight reduction might be a risk factor for regain of weight. (Arch Dis Child 1999;81:426-428)
\end{abstract}

Keywords: training programme; obesity; body composition

Department of Paediatrics, University of Graz, A-8036 Graz, Austria

J Schwingshandl

B Eibl

$S$ Wallner

M Borkenstein

Department of Sport Sciences, University of Graz

K Sudi

Correspondence to: Dr Schwingshandl. email: Josefs@Styria.co.at

Accepted 25 June 1999
Obesity is an ongoing problem in paediatric and adolescent health care. In previous work we identified reduction in fat free mass during rapid weight loss by energy reduction as a major factor for later regain in weight. ${ }^{1}$ Our present study aimed to: evaluate a standardised training programme for maintenance of fat free mass during weight reduction; and study the effect of changes in fat free mass during weight reduction on the long term outcome. Resistance training has been shown to be effective in increasing strength in children. ${ }^{2}$ There is limited information as to whether muscle growth (hypertrophy) can be induced in obese children.

\section{Subjects}

Thirty children consented to participate in the study and were assigned to two groups at random: group A (six boys, eight girls; mean (SD) age, 11.0 (2.5) years; mean standard deviation score for body mass index (BMI-SDS), 5.58 (2.46)) received standardised dietary advice for weight reduction by a dietitian at baseline and after four, eight, and 12 weeks of the study. In addition, subjects participated in a training programme twice weekly. Group B (seven boys, nine girls; mean age, 12.2 (2.7) years; mean BMI-SDS, 5.33 (1.79)) had the dietary intervention only.

\section{Methods}

BODY COMPOSITION

Body composition was estimated from bioelectrical impedance analysis. Measurements were performed at baseline and after four, eight, and 12 weeks. Total body resistance was measured by a bioelectrical impedance analyser (AkernRJL BIA 101/S) in supine position as described previously. ${ }^{1}$ Fat free mass was estimated from the resistance index (RI), height, and age of the subject using the equations given by Shaefer et al (fat free mass $(\mathrm{kg})=0.15+0.65 \times \mathrm{RI}+$ $0.68 \times$ age (years)). ${ }^{3}$ These equations have been established for the paediatric age range (3.919.3 years) by validating fat free mass predicted by bioelectric impedance analysis against a potassium dilution technique. However, these equations have not been evaluated for our population and no impedance predictions for Austrian children exist.

To assess the repeatability of our measurements, bioelectric impedance analysis was performed in 36 non-obese children with type I diabetes (22 boys, 14 girls; mean age, 12.3 years; SD, 2.6; range, 8.0-17.5) on two days spaced two weeks apart. The mean (SD) differences between the two measurements were 8.47 (30.44) ohms for resistance and 0.28 (1.64) $\mathrm{kg}$ for fat free mass.

\section{DIETARY INTERVENTION}

Both groups participated in the same dietary education programme. General dietary advice was given by group teaching. This included: energy requirements; relation of the different nutrients (protein, fat, and carbohydrates) in a balanced diet $(20 \% / 30 \% / 50 \%$ of total energy); and the importance of fibre, vitamins, minerals, and fluids.

Energy intake was restricted to $4180 \mathrm{~kJ} /$ day in both groups; children older than 14 years were restricted to $5016 \mathrm{~kJ} /$ day (girls) and $5852 \mathrm{~kJ} /$ day (boys). 
Table 1 Characteristics at baseline and after 12 weeks (group A)

\begin{tabular}{|c|c|c|c|c|c|c|c|c|}
\hline \multirow[b]{2}{*}{ Subject } & \multirow[b]{2}{*}{ Sex } & \multicolumn{4}{|l|}{ Initial } & \multicolumn{3}{|c|}{ After 12 weeks } \\
\hline & & Age (years) & Weight (kg) & $B M I-S D S$ & $F F M(k g)$ & Weight (kg) & $B M I-S D S$ & $F F M(\mathrm{~kg})$ \\
\hline 1 & M & 10.0 & 54.0 & 4.29 & 27.4 & 55.6 & 4.28 & 30.3 \\
\hline 2 & $\mathrm{~F}$ & 13.0 & 73.8 & 4.02 & 43.7 & 73.5 & 3.95 & 45.2 \\
\hline 3 & $\mathrm{~F}$ & 9.2 & 41.9 & 3.77 & 25.7 & 42.3 & 3.19 & 26.6 \\
\hline 4 & $\mathrm{~F}$ & 9.2 & 59.4 & 6.22 & 31.8 & 60.1 & 5.64 & 32.0 \\
\hline 5 & M & 12.6 & 59.5 & 2.78 & 36.5 & 61.2 & 2.89 & 40.5 \\
\hline 6 & $\mathrm{~F}$ & 11.6 & 64.5 & 4.75 & 32.1 & 62.3 & 4.04 & 32.4 \\
\hline 7 & M & 14.4 & 74.6 & 3.49 & 43.8 & 75.6 & 3.41 & 46.8 \\
\hline 8 & M & 10.6 & 53.5 & 3.45 & 33.0 & 52.5 & 2.78 & 34.2 \\
\hline 9 & $M$ & 13.7 & 68.6 & 4.88 & 41.8 & 68.7 & 4.30 & 44.7 \\
\hline 10 & $\mathrm{~F}$ & 6.4 & 47.0 & 11.52 & 22.1 & 46.9 & 10.95 & 22.0 \\
\hline 11 & M & 9.6 & 57.5 & 6.26 & 34.5 & 56.3 & 5.02 & 37.8 \\
\hline 12 & $\mathrm{~F}$ & 7.8 & 48.3 & 7.90 & 25.4 & 47.1 & 7.05 & 25.3 \\
\hline 13 & $\mathrm{~F}$ & 14.7 & 107.0 & 5.85 & 49.4 & 98.0 & 4.71 & 64.2 \\
\hline 14 & $\mathrm{~F}$ & 11.2 & 75.9 & 8.99 & 33.3 & 75.9 & 8.61 & 36.1 \\
\hline Mean & & 11.0 & 63.3 & 5.58 & 34.3 & 62.6 & 5.06 & 37.0 \\
\hline SD & & 2.5 & 16.5 & 2.46 & 8.0 & 14.8 & 2.34 & 10.9 \\
\hline
\end{tabular}

Paired comparisons $t$ test:

Weight change: mean -0.68 , SEM 0.71, p $=0.35$.

Change in BMI-SDS: mean -0.53 , SEM 0.11, p $=0.0003$

Change in fat free mass (FFM): mean 2.68, SEM 1.00, p $=0.02$.

Table 2 Characteristics at baseline and after 12 weeks (group B)

\begin{tabular}{|c|c|c|c|c|c|c|c|c|}
\hline \multirow[b]{2}{*}{ Subject } & \multirow[b]{2}{*}{ Sex } & \multicolumn{4}{|l|}{ Initial } & \multicolumn{3}{|c|}{ After 12 weeks } \\
\hline & & Age (years) & Weight (kg) & $B M I-S D S$ & $F F M(k g)$ & Weight (kg) & $B M I-S D S$ & $F F M(\mathrm{~kg})$ \\
\hline 1 & $M$ & 11.7 & 55.7 & 5.15 & 29.4 & 55.7 & 4.51 & 30.3 \\
\hline 2 & $\mathrm{~F}$ & 10.9 & 85.3 & 8.59 & 37.9 & 83.4 & 7.79 & 38.0 \\
\hline 3 & $\mathrm{~F}$ & 8.9 & 48.4 & 5.84 & 27.0 & 44.6 & 4.43 & 27.4 \\
\hline 4 & $M$ & 11.8 & 64.1 & 3.62 & 33.5 & 64.8 & 3.51 & 32.5 \\
\hline 5 & M & 11.7 & 84.0 & 7.15 & 40.3 & 83.5 & 6.77 & 40.6 \\
\hline 6 & $M$ & 12.1 & 58.6 & 4.99 & 32.1 & 59.6 & 4.64 & 33.2 \\
\hline 7 & $\mathrm{~F}$ & 12.9 & 73.2 & 5.31 & 41.4 & 75.4 & 5.47 & 43.9 \\
\hline 8 & M & 16.5 & 99.0 & 3.76 & 63.7 & 100.8 & 3.80 & 61.1 \\
\hline 9 & M & 11.8 & 53.1 & 3.54 & 32.7 & 54.6 & 3.63 & 33.4 \\
\hline 10 & M & 14.2 & 106.8 & 9.29 & 46.7 & 97.9 & 7.05 & 51.4 \\
\hline 11 & $\mathrm{~F}$ & 13.2 & 66.6 & 4.97 & 35.5 & 65.8 & 4.18 & 35.5 \\
\hline 12 & $\mathrm{~F}$ & 18.8 & 98.1 & 4.48 & 49.6 & 97.9 & 4.79 & 49.5 \\
\hline 13 & $\mathrm{~F}$ & 12.7 & 75.6 & 3.67 & 44.5 & 75.0 & 3.45 & 43.7 \\
\hline 14 & $\mathrm{~F}$ & 9.0 & 50.0 & 4.54 & 31.0 & 50.3 & 4.01 & 30.1 \\
\hline 15 & $\mathrm{~F}$ & 10.5 & 47.2 & 3.61 & 25.2 & 48.6 & 3.84 & 25.3 \\
\hline 16 & $\mathrm{~F}$ & 8.0 & 41.1 & 6.81 & 22.5 & 41.0 & 5.32 & 24.5 \\
\hline Mean & & 12.2 & 69.2 & 5.33 & 37.1 & 68.7 & 4.82 & 37.5 \\
\hline SD & & 2.7 & 20.6 & 1.79 & 10.5 & 19.7 & 1.33 & 10.2 \\
\hline
\end{tabular}

Paired comparisons $t$ test:

Weight change: mean -0.49 , SEM $0.67, \mathrm{p}=0.47$.

Change in BMI-SDS: mean -0.51, SEM 0.18, $\mathrm{p}=0.01$.

Change in fat free mass (FFM): mean 0.43, SEM 0.41, $\mathrm{p}=0.31$.

All participants received written information regarding an energy restricted, balanced diet, and detailed information of the daily allowance of each food component to avoid over consumption.

At each visit (at baseline; after four, eight, and 12 weeks; and after 6 and 12 months) each participant received dietary re-education on an individual basis.

TRAINING PROGRAMME

According to the recommendations of the committee on sports medicine, ${ }^{4}$ we established an individualised training programme. The use of proper technique and moderate intensity has been shown to be crucial in strength training with children. ${ }^{4}$ The use of lighter loads than those used by adults, ${ }^{5}$ and proper supervision and instruction should help to minimise the risk of injury during participation in resistance training. ${ }^{6}$

Training sessions took place twice weekly in a public gym, with a duration of approximately 60-70 minutes for each session. After a warm up period on either a stationary bike, treadmill, or rowing ergometer, and a light stretch, each child performed two sets out of the following exercises: lying leg press; seated leg extensions and leg curls; seated bench press; lat pulldown to the front and long pulley row; seated shoulder press; triceps pushdowns and triceps extension at the dips machine; dumbbell biceps curls; calf raises seated and crunches. All exercises except dumbbell biceps curls were done on weightstacking machines (Galaxy-Sport, Carspach, France), in which resistance can be increased gradually.

The volume (number of sets performed during training sessions) increased after two weeks, working up to as many as three to four sets for each exercise. Intensity, expressed as a " 10 repetition maximum" (10 RM) - the maximum load the child could perform 10 repeats with-was adapted during the time course of strength gain. Sets were pyramided: starting with a light set ( $50 \%$ of the $10 \mathrm{RM})$, with each following set using a slightly higher load. Only the last set was performed until muscle failure. 


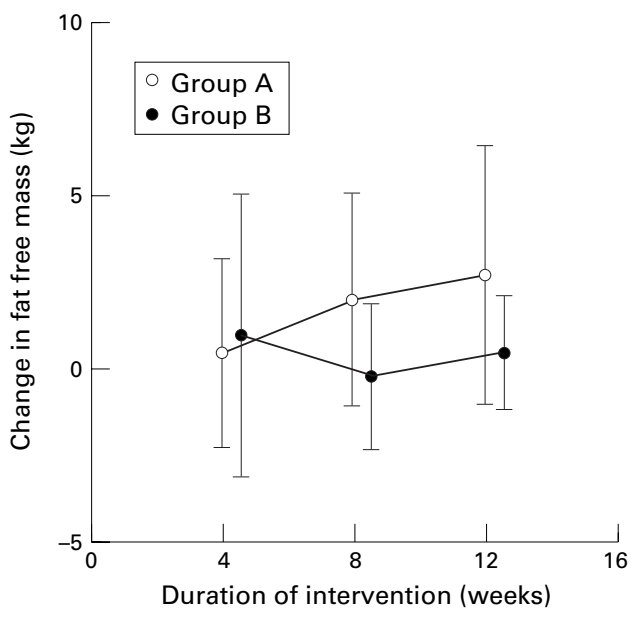

Figure 1 Changes in fat free mass (mean and SD) during the first 12 weeks.

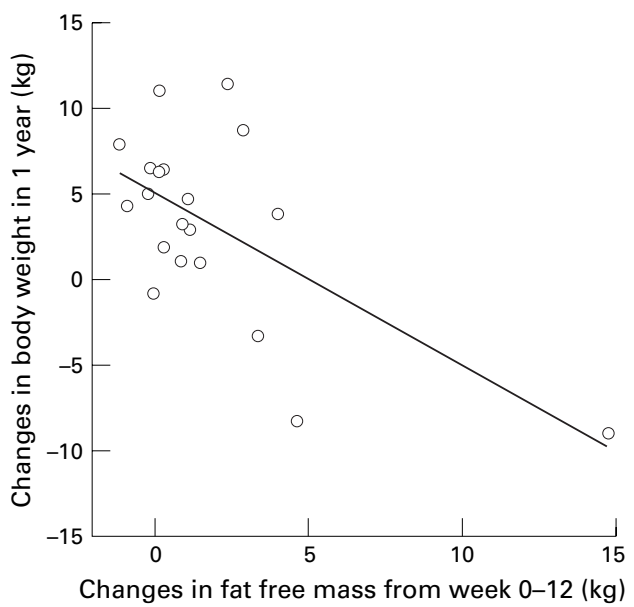

Figure 2 Effect of changes in fat free mass on change in body weight after one year.

If the child was able to perform more than 10 repetitions in the final set, the resistance was increased for the next training session.

\section{STATISTICAL ANALYSIS}

Statistical analysis was performed using the software package SAS 6.10 for Windows (SAS Institutes, Cary, North Carolina, USA). Methods used were paired comparisons $t$ test, Wilcoxon rank sum test, and Spearman's correlation coefficient. $\mathrm{p}$ Values of 0.05 or less were considered significant.

\section{ETHICAL CONSIDERATIONS}

All patients and one parent gave fully informed written consent to participate in our study. The study was approved by the local ethics committee.

\section{Results}

Tables 1 and 2 give patients' characteristics at baseline and after 12 weeks. In group A, changes in BMI-SDS and fat free mass were significant, whereas weight change was not significant. In group $\mathrm{B}$ only BMI-SDS changed significantly, whereas changes in weight and fat free mass were not significant. The mean (SD) change in fat free mass was significantly different between the two groups (group A, 2.68 (3.74) kg; group B, 0.43 (1.65) kg; p = 0.015) (fig 1).

The change in fat free mass after 12 weeks was inversely correlated with changes in body weight after one year $(r=-0.44 ; \mathrm{p}=0.05)$, as assessed in the 20 subjects (fig 2 ).

\section{Discussion}

We found that a standardised resistance training programme adapted for obese children and adolescents significantly increased percentage fat free mass during weight reduction when compared with energy reduction only. An increase in fat free mass during weight reduction was weakly associated with a better long term outcome with respect to body weight.

Exercise has been part of most weight reduction programmes for obese children and adolescents. ${ }^{8}$ However, exercise mostly consisted of aerobic exercise such as swimming, jogging, playing soccer, etc. Those types of exercise increase energy expenditure during exercise but have no or little effect on resting energy expenditure. A reduction in resting energy expenditure after weight loss is the main risk factor for later regain of weight. ${ }^{9-11}$ Resting energy expenditure is closely related to fat free mass in obese children. ${ }^{12}$ Therefore, our study aimed to prevent reduction in resting energy expenditure after weight loss by a training programme focused on increasing fat free mass. Indeed, this training did succeed in increasing fat free mass during weight reduction.

In summary, our study indicates that a weight reduction programme accompanied by a training programme focusing on increasing fat free mass should be investigated further in an attempt to improve the long term outcome of childhood and adolescent obesity.

We thank the "Fit For Life" Gym in Graz for providing the equipment during the training period.

1 Schwingshandl J, Borkenstein M. Changes in lean body mass in obese children during a weight reduction program: effect on short term and longterm outcome. Int $\mathcal{F}$ Obes 1995;19:752-5

2 Blimkie CJR. Resistance training during pre- and early puberty: efficacy, trainability, mechanisms, and persistence. Can $\mathcal{F}$ Sport Sci 1992;17:264-79.

3 Schaefer F, Georgi M, Zieger A, Schärer K. Usefulness of bioelectrical impedance and skinfold measurements in predicting fat-free mass derived from total body potassium in children. Pediatr Res 1994;35:617-24.

4 Committee on Sports Medicine. Strength training, weight and power lifting, and body building by children and adoand power lifting, and body buildin

5 Kraemer WJ, Fry AC, Frykman PN, Conroy B, Hoffman J. Resistance training and youth. Pediatric Exercise Science 1989;1:336-50

6 Les Sewall BS, Micheli LJ. Strength training for children. $\mathcal{F}$ Pediatr Orthop 1986;6:143-6.

7 Schlotzhauer SD, Littell RC. SAS system for elementary statistical analysis. North Carolina: SAS Institute, 1987.

8 Dietz WH. Therapeutic strategies in childhood obesity. Horm Res 1993;39(suppl 3):86-90.

9 Bessard T, Schutz Y, Jequier E. Energy expenditure and postprandial thermogenesis in obese women before and after weight loss. Am F Clin Nutr 1983;38:680-93.

10 Ravussin E, Burnand B, Schutz Y, Jequier E. Energy expenditure before and during energy restriction in obese patients. Am f Clin Nutr 1985;41:753-9.

11 Ravussin E, Lillioja S, Knowler WC, et al. Reduced energy expenditure as a risk factor for body weight gain. $N$ Engl 7 Med 1988;318:467-72.

12 Zwiauer KFM, Müller T, Widhalm K. Resting metabolic rate in obese children before, during and after weight loss. Int $\mathcal{F}$ Obes 1992;16:11-16. 